\title{
Protective Effect of Tetrandrine in a Rabbit Model of Osteoarthritis
}

\author{
Fengfeng WU, Juntao XU, ${ }^{1}$ Zaihua $\mathrm{ZHU}^{3}$ \\ ${ }^{1}$ Department of Orthopaedic Surgery, Huzhou Central Hospital, Huzhou, China \\ ${ }^{2}$ Department of Orthopaedic Surgery, The Traditional Chinese Hospital of Huzhou, Huzhou, China \\ ${ }^{3}$ Department of Rheumatology, The First Affiliated Hospital of Wenzhou Medical University, Wenzhou, China
}

\begin{abstract}
Objectives: This study aims to investigate the antiinflammatory effect of tetrandrine in a rabbit model of osteoarthritis.

Materials and methods: The cruciate ligaments and medial menisci of 36 female New Zealand white rabbits (each weighing $2.0-3.0 \mathrm{~kg}$ ) were resected. Six weeks after surgery, the animals were randomly divided into three groups ( $\mathrm{n}=12 \mathrm{in}$ each): tetrandrine ( $20 \mathrm{mg} / \mathrm{kg} / \mathrm{day})$, indomethacin ( $3 \mathrm{mg} / \mathrm{kg} / \mathrm{day}$ ) and control (saline $10 \mathrm{~mL} /$ day) groups. After 14 days of treatment, the results were assessed by macroscopic observation, histological evaluation, measurement of the levels of interleukin-1 beta, tumor necrosis factor-alpha and nitric oxide in the synovial fluid and messenger ribonucleic acid expression of metalloproteinase- 3 in synovia.

Results: In the tetrandrine group, the pathological changes were more attenuated than in the control group. Levels of interleukin-1 beta, tumor necrosis factor-alpha, nitric oxide and metalloproteinase-3 in the synovial fluid or synovium were all significantly suppressed in the tetrandrine group compared with the control group. There were no significant differences between the tetrandrine and indomethacin groups.

Conclusion: The results of this study demonstrate that tetrandrine may protect against the development of experimentally induced osteoarthritis. Keywords: Osteoarthritis; rabbit model; tetrandrine.
\end{abstract}

Osteoarthritis $(\mathrm{OA})$ is characterized by progressive cartilage degeneration that can ultimately lead to a complete loss of joint cartilage. Although OA is classified as a non-inflammatory arthropathy, inflammation has been shown to play a significant role in disease progression. Several studies have shown that cartilage destruction in $\mathrm{OA}$ is the result of the activity of cytokines, such as interleukin$1 \beta$ (IL-1 $\beta$ ), tumor necrosis factor-alpha (TNF- $\alpha$ ), prostaglandins (PGs), nitric oxide (NO), proteases and other inflammatory mediators. ${ }^{1-3}$

Tetrandrine is a major bisbenzylisoquinoline alkaloid isolated form Stephania tetrandra S. Moore, which has been used for 2,000 years in China as an antirheumatic herbal drug. Similar to standard drugs, tetrandrine inhibits the production and activation of ILs, TNF- $\alpha$,
PGs, cycloxygenase-2 (COX-2) and T cells under experimental conditions to reduce inflammation..$^{4-6}$ However, most of the previous studies have focused on rheumatoid arthritis. Therefore, in this study, we aimed to investigate the antiinflammatory effect of tetrandrine in a rabbit model of OA.

\section{MATERIALS AND METHODS}

Thirty-six female New Zealand white rabbits (each weighing $2.0-3.0 \mathrm{~kg}$ ) were included in the study, which was conducted between February 2016 and June 2016 at Wenzhou Medical University. Animal experiments were performed in accordance with internationally accredited guidelines and approved by the local laboratory's Institutional Animal Care

Received: February 10, 2017 Accepted: April 04, 2017 Published online: September 13, 2017

Correspondence: Zaihua Zhu, MD. Department of Rheumatology, The First Affiliated Hospital of Wenzhou Medical University, 325000 Wenzhou, China.

Tel: +86-577-88069252 e-mail:9094116@qq.com

(2018 Turkish League Against Rheumatism. All rights reserved. 
and Use Committee. After anesthesia with 10\% chloral hydrate $(4 \mathrm{~mL} / \mathrm{kg})$, the cruciate ligaments and medial menisci were resected using the Hulth technique to establish OA models. ${ }^{7}$ Following recovery from anesthesia, all animals were allowed to be freely active within their cages. Six weeks after the surgery, the animals were divided into three groups ( $\mathrm{n}=12$ in each): tetrandrine (20 $\mathrm{mg} / \mathrm{kg} /$ day), indomethacin (3 $\mathrm{mg} / \mathrm{kg} /$ day) and control (saline $10 \mathrm{~mL} /$ day) groups. During the next 14 days, the rabbits were observed daily to monitor their health status.

After 14 days of treatment, the rabbits were sacrificed and the femoral condyles were collected for observation. After macroscopic examination, isolated samples were fixed with 10\% buffered formalin, embedded in paraffin, and decalcified with $20 \%$ ethylenediaminetetraacetic acid solution for further histological evaluation. Serial sections $(5 \mu \mathrm{m})$ through the entire specimen were prepared and stained with hematoxylin and eosin. The severity of the osteoarthritic lesions was graded on a scale of $0-14$ by three independent observers using the Mankin scoring system as follows: Mankin score 0, normal cartilage with a smooth surface and a regular zonal distribution of chondrocytes; Mankin score 1-4, cartilage surface shows fibrillations and a superficial loss of proteoglycans, but the zonal structure is intact; Mankin score 5-8, cartilage samples have clefts reaching down to the middle cartilage zone, and clusters of proliferating chondrocytes are present; and Mankin score $\geq 9$, severely affected cartilage samples with clefts reaching down to the deep zone, in which the tangential zone is lost and chondrocyte clusters are present. ${ }^{8}$

Immediately after the rabbits were sacrificed, the synovial fluid (SF) was aspirated from the affected joints. The levels of IL- $1 \beta$ and TNF- $\alpha$ in the SF were measured using enzyme-linked immunosorbent assay kits (Shenggong Company, Shanghai, China), and all assays were performed according to the manufacturer's instructions. NO was detected using the Griess method. ${ }^{9}$

After sacrifice, synovia of the operated knee was harvested for measurement of messenger ribonucleic acid (mRNA) expression of metalloproteinase-3 (MMP-3). Primers were designed and synthesized. Total RNA was extracted and amplified by reverse transcription polymerase chain reaction (Shenggong Company, Shanghai, China). The relative levels of mRNAs were normalized to the mean gray value of $\beta$-actin and then expressed as relative mRNA value for each specimen.

\section{Statistical analysis}

Statistical analysis was carried out using SPSS 16.0 software package (SPSS Inc., Chicago, IL, USA). Data were expressed as mean \pm standard deviation. Differences between groups were compared using Student's t-test and the level of significance was set at $p<0.05$.

\section{RESULTS}

In the control group, the cartilage of the femoral condyles appeared to have significant defects including osteophytes. In the tetrandrine and indomethacin groups, cartilage lesions associated with OA were clearly seen, but the severity was milder than in the control group.

Specimens from the control group showed morphologic changes characteristic of OA. These changes included disarranged chondrocytes with degeneration or necrosis, ulcerations in the deep cartilage layer, and fibroplasia at the bottom of the ulcers (Figure 1a). In both the indomethacin and tetrandrine groups, histological changes associated with OA were clearly seen, but the severity was milder than in the control group (Figures $1 \mathrm{~b}$ and c). Mankin scores in the indomethacin $(2.94 \pm 0.98)$ and tetrandrine $(2.85 \pm 0.74)$ groups were significantly lower than in the control group $(6.45 \pm 1.23)$ $(p<0.05)$, as seen in Figure 2. However, no significant differences between the indomethacin and tetrandrine groups were observed.

As shown in Figures 3 and 4 , the levels of IL-1 $\beta$, TNF- $\alpha$ and NO in the SF were significantly decreased in the indomethacin and tetrandrine groups compared with the control group $(p<0.05)$, with no significant differences between the indomethacin and tetrandrine groups ( $\mathrm{p}>0.05)$.

There was a significant decrease in mRNA expression of MMP-3 in the indomethacin and tetrandrine groups compared to the control group $(p<0.05)$. However, there was no significant difference between the indomethacin and tetrandrine groups ( $p>0.05)$ (Figure 5). 

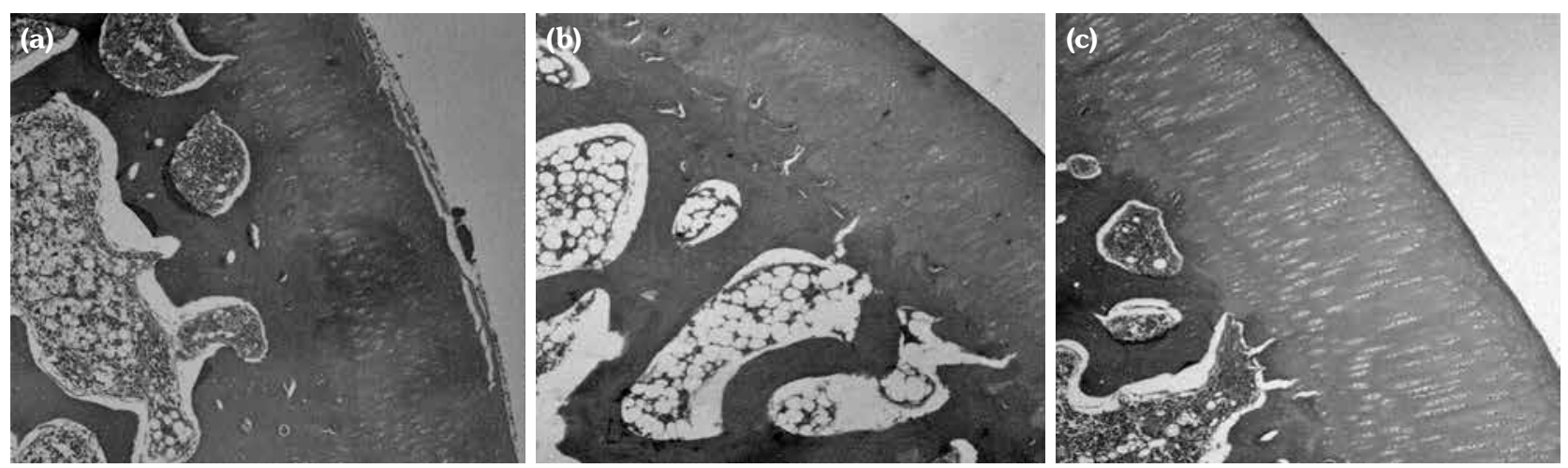

Figure 1. Histological findings of femoral condyles (a) in control, (b) indomethacin and (c) tetrandrine groups (H-Ex400).

\section{DISCUSSION}

Osteoarthritis, the most common cause of pain and disability in the elderly, is a complex disease characterized by bone remodeling, synovial inflammation, and cartilage loss. Generally, OA is an articular cartilage injury caused by an imbalance in the degradation and synthesis of chondrocytes, extracellular matrix, and subchondral bone. Studies have confirmed that several biological factors are involved in the development of $\mathrm{OA}$, including IL-1 $\beta$, TNF- $\alpha$, MMPs and NO. ${ }^{1-3}$

Non-surgical modalities for OA include weight loss, exercise, non-steroidal antiinflammatory drugs, analgesics, and intra-articular hyaluronic acid [AU: correct?]. ${ }^{10}$ Since inflammation has been shown to play a significant role in OA progression, there have been several studies in animal models of $\mathrm{OA}$ regarding the use of antiinflammatory drugs for treatment. IL-1 receptor antagonists (anakinra),

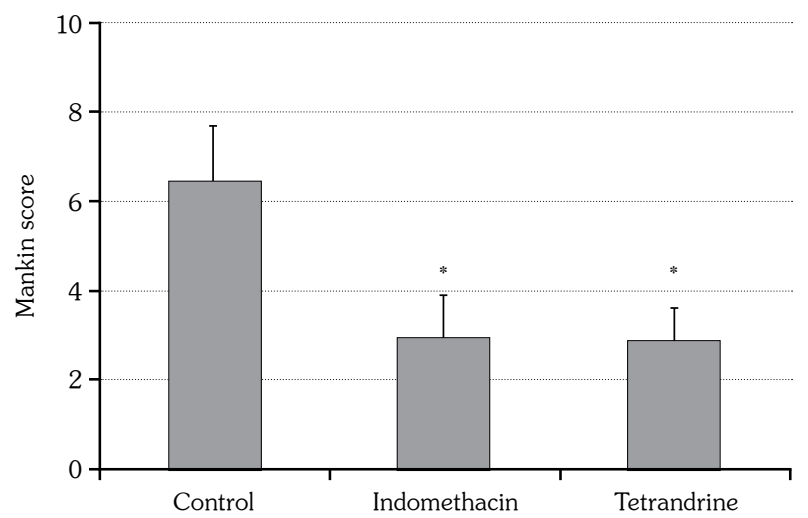

Figure 2. Histological scores of cartilage according to Mankin scoring system. * $\mathrm{p}<0.05$ compared to control group.
anti-TNF- $\alpha$ monoclonal antibody (infliximab) and selective COX-2 inhibitor (celecoxib) have all shown significant therapeutic effects. ${ }^{11-13}$

In this study, we found that the levels of IL-1 $\beta$, TNF- $\alpha$, NO and MMP-3 in the SF or synovia were all significantly suppressed in the tetrandrine group compared with the control group. In the tetrandrine group, the Mankin score in the articular cartilage improved significantly compared with the control group. This indicates that tetrandrine might protect against early osteoarthritic cartilage damage by delaying cartilage degeneration, as was also shown by the results of our macroscopic and histological analyses. However, since there was no significant difference in the two active treatment groups, it is expected that tetrandrine would have the same protective effect on articular cartilage, as indomethacin in a rabbit OA model.

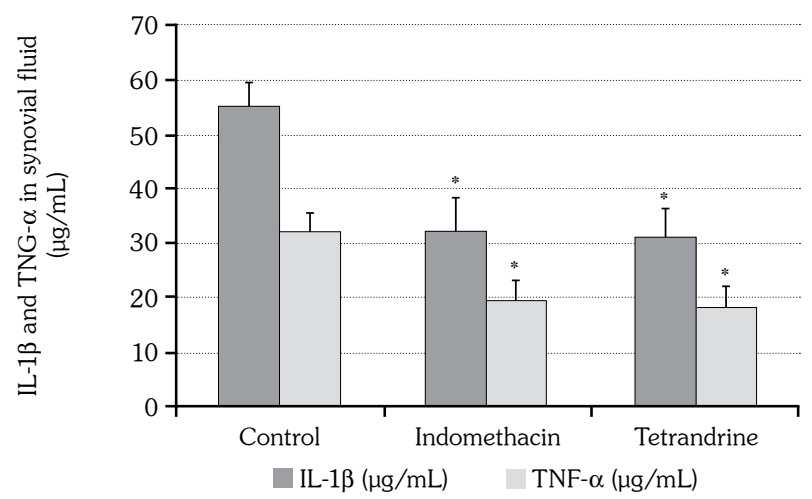

Figure 3. Contents of interleukin- $1 \beta$ and tumor necrosis factor-alpha in synovial fluid. * $p<0.05$ compared to control group; IL-1 $\beta$ : Interleukin-1 $\beta$; TNF- $\alpha$ : Tumor necrosis factor-alpha. 


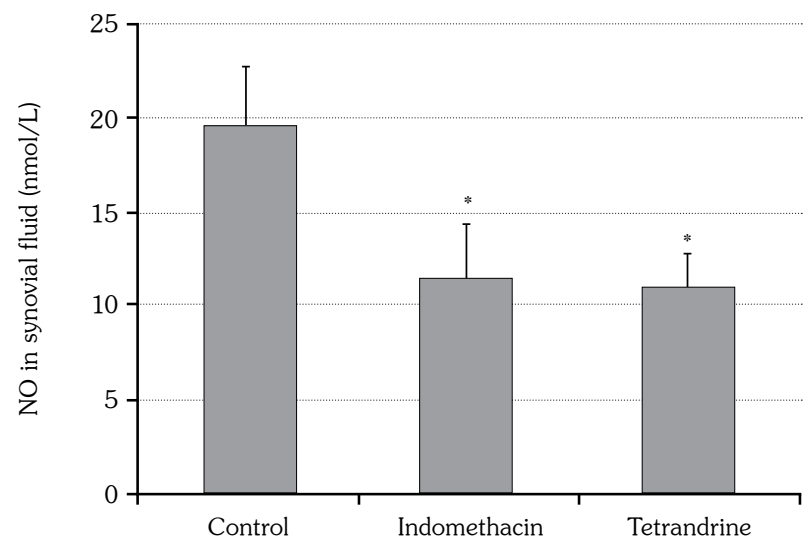

Figure 4. Contents of nitric oxide in synovial fluid. * $p<0.05$ compared to control group. NO: Nitric oxide.

There may be two major possible reasons for these findings. First, IL-1 $\beta$ is a cytokine involved in the processes of cartilage degradation. In response to IL-1 $1 \beta$, chondrocytes secrete TNF- $\alpha$, neutral MMPs and NO. IL- $1 \beta$ and TNF- $\alpha$ localize with increased levels of MMPs, and IL1 $\beta$ has been shown to up-regulate MMP expression in normal human chondrocytes. ${ }^{14,15}$ Tetrandrine may directly inhibit IL-1 $\beta$ and TNF- $\alpha$ expression by blocking the nuclear translocation of nuclear factor kappa B. ${ }^{13,16}$ Second, tetrandrine may suppress articular inflammatory response by inhibiting COX-2 inactivation. ${ }^{6,17} \mathrm{COX}-2$ induction was observed in the chondrocytes of $\mathrm{OA}$ joints, and this produces PGs. PG E2 has been shown to accelerate cartilage degeneration by inhibiting biosynthesis of glycosaminoglycans. ${ }^{18,19}$ Selective COX-2 inhibitors are effective for OA treatment because they attenuate synovial inflammation and cartilage destruction. Blocking PG E2 prevents cytokine-induced cartilage damage and increases synthesis of glycosaminoglycans in OA cartilage.

There are several limitations in our study. First, the occurrence of osteoarthritis was evaluated at only one time point, 14 days after the administration. Therefore, it is possible that osteoarthritis had been repaired before this time point or that the development of osteoarthritis was delayed. Second, we limited the intake of tetrandrine to $20 \mathrm{mg} / \mathrm{kg} /$ day diet, since we were unable to determine the optimum level of tetrandrine that can suppress the development of osteoarthritis. Thus, future studies should

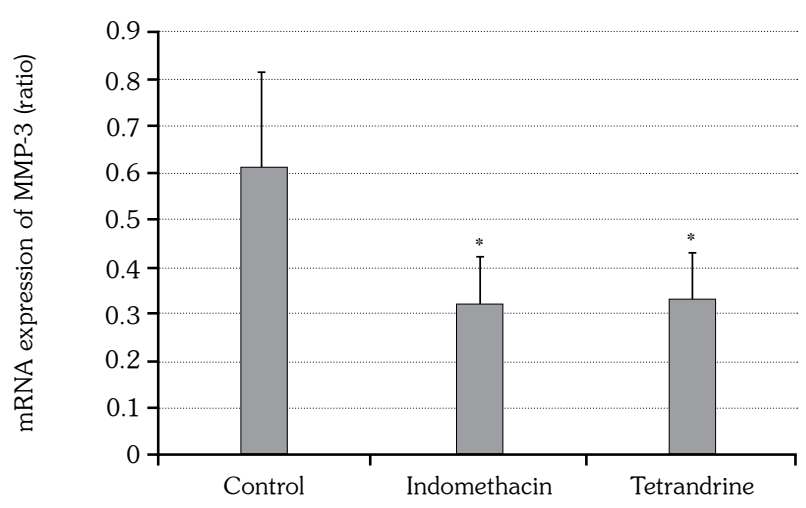

Figure 5. Messenger ribonucleic acid expression of metalloproteinase -3 in synovia. ${ }^{*} p<0.05$ compared to control group; mRNA: Messenger ribonucleic acid; MMP-3: Metalloproteinase-3.

determine the timing of osteoarthritis development and the level of tetrandrine required to elicit this suppressive effect on the development.

In conclusion, our results suggested that tetrandrine may have a potential therapeutic role in the management of $\mathrm{OA}$ due to its inhibition of the IL-1 $\beta$-induced release of TNF- $\alpha$, MMP- 3 and NO. This novel therapeutic strategy may be a promising treatment option for OA.

\section{Declaration of conflicting interests}

The authors declared no conflicts of interest with respect to the authorship and/or publication of this article.

\section{Funding}

The authors received no financial support for the research and/or authorship of this article.

\section{REFERENCES}

1. Kapoor M, Martel-Pelletier J, Lajeunesse D, Pelletier $\mathrm{JP}$, Fahmi H. Role of proinflammatory cytokines in the pathophysiology of osteoarthritis. Nat Rev Rheumatol 2011;7:33-42.

2. Loeser RF. Molecular mechanisms of cartilage destruction in osteoarthritis. J Musculoskelet Neuronal Interact 2008;8:303-6.

3. Lapaj L, Markuszewski J, Wierusz-Kozlowska M. Current views on the pathogenesis of osteoarthritis. Chir Narzadow Ruchu Ortop Pol 2010;75:248-60. [Abstract]

4. Choi HS, Kim HS, Min KR, Kim Y, Lim HK, Chang YK, et al. Anti-inflammatory effects of fangchinoline and tetrandrine. J Ethnopharmacol 2000;69:173-9. 
5. Zhou X, Li W, Jiang L, Bao J, Tao L, Li J, et al. Tetrandrine Inhibits the Wnt/ $\beta$-Catenin Signalling Pathway and Alleviates Osteoarthritis: An In Vitro and In Vivo Study. Evid Based Complement Alternat Med 2013;2013:809579.

6. Wu SJ, Ng LT. Tetrandrine inhibits proinflammatory cytokines, iNOS and COX-2 expression in human monocytic cells. Biol Pharm Bull 2007;30:59-62.

7. Hulth A, Lindberg L, Telhag H. Experimental osteoarthritis in rabbits. Preliminary report. Acta Orthop Scand 1970;41:522-30.

8. van der Sluijs JA, Geesink RG, van der Linden AJ, Bulstra SK, Kuyer R, Drukker J. The reliability of the Mankin score for osteoarthritis. J Orthop Res 1992;10:58-61.

9. Green LC, Wagner DA, Glogowski J, Skipper PL, Wishnok JS, Tannenbaum SR. Analysis of nitrate, nitrite, and [15N]nitrate in biological fluids. Anal Biochem 1982;126:131-8.

10. McAlindon TE, Bannuru RR, Sullivan MC, Arden NK, Berenbaum F, Bierma-Zeinstra SM, et al. OARSI guidelines for the non-surgical management of knee osteoarthritis. Osteoarthritis Cartilage 2014;22:363-88.

11. Frisbie DD, Ghivizzani SC, Robbins PD, Evans $\mathrm{CH}$, McIlwraith $\mathrm{CW}$. Treatment of experimental equine osteoarthritis by in vivo delivery of the equine interleukin-1 receptor antagonist gene. Gene Ther 2002;9:12-20

12. Zhang Q, Lv Hh, Chen A, Liu F, Wu X. Efficacy of infliximab in a rabbit model of osteoarthritis. Connect Tissue Res 2012;53:355-8.

13. Jiang D, Zou J, Huang L, Shi Q, Zhu X, Wang G, et al. Efficacy of intra-articular injection of celecoxib in a rabbit model of osteoarthritis. Int $\mathrm{J}$ Mol Sci 2010;11:4106-13.

14. Daheshia M, Yao JQ. The interleukin 1beta pathway in the pathogenesis of osteoarthritis. J Rheumatol 2008;35:2306-12.

15. Goldring SR, Goldring MB. The role of cytokines in cartilage matrix degeneration in osteoarthritis. Clin Orthop Relat Res 2004;427:27-36.

16. Gao LN, Feng QS, Zhang XF, Wang QS, Cui YL. Tetrandrine suppresses articular inflammatory response by inhibiting pro-inflammatory factors via NF-кB inactivation. J Orthop Res 2016;34:1557-68.

17. Bhagya N, Chandrashekar KR. Tetrandrine-A molecule of wide bioactivity. Phytochemistry 2016;125:5-13.

18. El Hajjaji H, Marcelis A, Devogelaer JP, Manicourt $\mathrm{DH}$. Celecoxib has a positive effect on the overall metabolism of hyaluronan and proteoglycans in human osteoarthritic cartilage. $\mathrm{J}$ Rheumatol 2003;30:2444-51.

19. Mastbergen SC, Bijlsma JW, Lafeber FP. Selective COX-2 inhibition is favorable to human early and latestage osteoarthritic cartilage: a human in vitro study. Osteoarthritis Cartilage 2005;13:519-26. 